\title{
Cenário da segurança alimentar e nutricional de famílias residentes no município de belém durante a pandemia de covid - 19 associada à medidas de isolamento social, políticas públicas e grupos de risco
}

\section{Food and nutritional security scenario for families resident in the city of belém during the covid pandemy - 19 associated with social isolation measures, public policies and risk groups}

DOI: $10.46919 / \operatorname{arch} v 2 \mathrm{n} 3-037$

Recebimento dos originais: 01/01/2021

Aceitação para publicação: 31/03/2021

\author{
Marcela Brito Parente \\ Faculdade UNINASSAU \\ Tv. Quintino Bocaiúva, 1808 - Nazaré, Belém - PA, 66035 - 190, Brasil \\ E-mail marcelabrito1999@outlook.com \\ Karla Cavalcante Quadros \\ Faculdade UNINASSAU \\ Tv. Quintino Bocaiúva, 1808 - Nazaré, Belém - PA, 66035 - 190, Brasil \\ E-mail kcavalcante915@gmail.com \\ Natália Pereira de Oliveira \\ Faculdade UNINASSAU \\ Tv. Quintino Bocaiúva, 1808 - Nazaré, Belém - PA, 66035 - 190, Brasil \\ E-mail nanaper.oliveira@gmail.com
}

\section{RESUMO}

A pandemia de Covid - 19 trouxe consigo diversas problemáticas que repercutiram negativamente na vida das pessoas e que agravaram o quadro de insegurança alimentar já instalado, com aumento do desemprego, restrições para o trabalho informal, o medo de ser infectado ao sair de casa, a suspensão das aulas, entre outros. Assim, a pesquisa buscou verificar o cenário da segurança alimentar e nutricional de famílias residentes no município de Belém durante a pandemia de Covid - 19 associada à medidas de isolamento social, políticas públicas e grupos de risco. Foi realizado um estudo transversal, quantitativo, com aplicação de um questionário online com 10 itens divulgado de 4 a 12 de outubro de 2020 em redes sociais (Whatsapp, Instagram e Facebook) com itens que abordavam a respeito do cenário de segurança alimentar durante a pandemia de Covid - 19 baseado na EBIA (Escala Brasileira de Insegurança Alimentar) com adapção para o referido período, além de insegurança alimentar ocasionada por medidas de isolamento, opinião a respeito do Auxílio Emergencial instituido pelo governo federal e de pertencer à algum grupo de risco da saúde e contou com a participação de 200 membros de cada família. De acordo com os resultados obtidos boa parte dos participantes durante a pandemia se encontravam em algum grau de insegurança alimentar, com ênfase naqueles que não possuiam uma alimentação saudável e variada e com maior percentual entre os maiores de 18 anos (32,4\%) além de menores de 18 anos que consumiram uma quantidade insuficiente de comida $(10,8 \%)$, no mais, a maioria considera que Auxílio Emergencial os ajudou a manter uma alimentação adequada (64,9\%), ainda, 29,7\% deixaram de ir ao mercado de modo a comprometer a sua alimentação, bem como a maioria possuia algum membro da família pertencente ao grupo de risco $(83,8 \%)$. Assim é possível concluir que é de fundamental importância o conhecimento a respeito do cenário de segurança alimentar da população para que possam ser tomadas medidas que visem combater os flagelos das 
desigualdades sociais e garantir a segurança alimentar e nutricional como forma de proteger as pessoas na pandemia especialmente as minorias e grupos de risco que tendem a estar mais vulneráveis nesses momentos de crise, além de fortalecer as políticas já existentes que visam minimizar tais desigualdades.

Palavras chave: Pandemia Covid - 19; Segurança Alimentar e Nutricional; Questionário; Escala Brasileira de Insegurança Alimentar e Nutricional; Auxílio Emergencial; Medidas de isolamento; Grupos de risco.

\begin{abstract}
The Covid - 19 pandemic brought with it several problems that had negative repercussions on people's lives and that aggravated the already established food insecurity framework, with increased unemployment, restrictions on informal work, the fear of being infected when leaving home, the suspension of classes, among others. Thus, the research sought to verify the food and nutritional security scenario of families living in the municipality of Belém during the Covid pandemic - 19 associated with measures of social isolation, public policies and risk groups. A cross-sectional, quantitative study was carried out, with the application of an online questionnaire with 10 items released from October 4th to 12th, 2020 on social networks (Whatsapp, Instagram and Facebook) with items that addressed the food safety scenario during the pandemic de Covid - 19 based on the EBIA (Brazilian Scale of Food Insecurity) with adaptation for the referred period, in addition to food insecurity caused by isolation measures, opinion regarding the Emergency Aid instituted by the federal government and belonging to some risk group of health and had the participation of 200 members of each family. According to the results obtained, most of the participants during the pandemic were in some degree of food insecurity, with emphasis on those who did not have a healthy and varied diet and with the highest percentage among those over 18 years old (32.4\%). of children under 18 years old who consumed an insufficient amount of food (10.8\%), moreover, most consider that Emergency Assistance helped them to maintain adequate nutrition (64.9\%), yet 29.7\% stopped go to the market in order to compromise their diet, as well as the majority had a family member belonging to the risk group $(83.8 \%)$. Thus, it is possible to conclude that knowledge about the population's food security scenario is of fundamental importance so that measures can be taken to combat the scourges of social inequalities and ensure food and nutritional security as a way to protect people from the pandemic, especially the minorities and groups at risk that tend to be more vulnerable in these moments of crisis, in addition to strengthening existing policies that aim to minimize such inequalities.
\end{abstract}

Key words: Covid Pandemic - 19, Food and nutrition security, Quis, Brazilian Scale of Food and Nutritional Insecurity, Emergency Assistance, Isolation measures, Groups of risk.

\title{
1 INTRODUÇÃO
}

A segurança alimentar e nutricional consiste em um direito que abrange o acesso aos alimentos em quantidade suficiente e com qualidade, podendo ser alcançada a partir de políticas e programas para promover, quer seja na dimensão individual ou coletiva, uma alimentação ideal e permanente (VASCONCELLOS e MOURA, 2018). Somado a isso, a presença de uma má alimentação e nutrição é capaz de gerar desigualdades, explicada através de quadros contraditórios dados os cenários de desnutrição e obesidade (PINHEIRO e CARVALHO, 2010).

A pandemia do Covid-19 deu maior visibilidade para as diferentes realidades presentes no país, expondo uma enorme parte da população que vive em contextos de vulnerabilidade social, principalmente no momento atual onde as taxas de desemprego subiram (RIBEIRO-SILVA, et al., 2020). Dessa forma, 
Rigaud et al.(2021) explica que o contexto de pandemia ao qual o Brasil enfrenta aumentou de forma exponencial a pobreza e a fome.

Ademais, os grupos de risco de pessoas mais propensas para a evolução do vírus são idosos, hipertensos, cardiopatas, diabéticos, portadores de doenças inflamatórias crônicas ou imunodepressoras, entre outros, porém conforme o avanço da doença os sintomas também foram agravantes em pessoas jovens (MINUSSI, et al., 2020). Somado a isso, o fortalecimento do sistema imunológico é fundamental para o enfrentamento da Covid-19 e isso é alcançado através de hábitos saudáveis como a ingestão constante de uma alimentação balanceada em nutrientes, dentre os mais importantes estão a vitamina C e D e o zinco (MINUSSI, et al., 2020).

Diante do isolamento social como medida sanitária, a economia entrou em crise, tanto pelo distanciamento, como pela desaceleração econômica, e para minimizá-la algumas medidas foram tomadas, no Brasil, estabeleceu-se o auxílio emergencial visando a proteção de trabalhadores informais, microempreendedores individuais, autônomos e desempregados (ALPINO, et al., 2020). No entanto, o auxílio emergencial reflete um caráter pejorativo e não da forma como deveria ser visto, que no caso, como dever do Estado, além de exclui grupos não inscritos no CadÚnico, como é o caso dos agricultores familiares, colocando em risco a segurança alimentar dessa população (MENEZES e BURITY, 2020; PICHONELLI, 2020).

\footnotetext{
A Escala Brasileira de Insegurança Alimentar (EBIA) visa mensurar de maneira direta a segurança alimentar e nutricional a partir da percepção e experiência da fome, porém graças ao número alto de questões e custos, utiliza-se em alguns estudos nacionais a escala americana que possui apenas seis questões (SANTOS, et al., 2014). Diante disso, a insegurança alimentar vai desde o sentimento de angústia e incerteza de ter condições de adquirir alimentos até a falta dele (BRASIL, 2006).
}

\section{OBJETIVOS}

2.1 Objetivo geral: Avaliar o cenário de segurança alimentar de famílias residentes no município de Belém durante a pandemia de Covid - 19 associada à medidas de isolamento social, políticas públicas e grupos de risco.

\subsection{Objetivos específicos:}

- Avaliar o cenário de segurança alimentar de famílias residentes no município de Belém durante a pandemia de Covid - 19;

- Avaliar se as medidas de isolamento social influenciaram na aquisição de alimentos e se houveram impactos negativos na alimentação;

- Avaliar se o Auxílio Emergencial instituido pelo Governo Federal consistia em significante política para assegurar a segurança alimentar e nutricional;

- Verificar se em cada família havia pelo menos uma pessoa que pertencia ao grupo de risco. 


\section{METODOLOGIA}

Foi realizado um estudo transversal, quantitativo, com aplicação de um questionário online com 10 itens divulgado de 4 a 12 de outubro de 2020 em redes sociais (Whatsapp, Instagram e Facebook) com itens que abordavam a respeito do cenário de segurança alimentar durante a pandemia de Covid - 19 baseado na EBIA (Escala Brasileira de Insegurança Alimentar) com adapção para o referido período, além de insegurança alimentar ocasionada por medidas de isolamento, opinião a respeito do Auxílio Emergencial instituido pelo governo federal e de pertencer à algum grupo de risco da saúde e contou com a participação de 200 membros de cada família. Os critérios de inclusão para participar da pesquisa eram: ter idade igual ou maior que 18 anos, ser membro de uma família e residir junto à esta em Belém no momento da pesquisa. Critérios de exclusão: ter idade inferior a 18 anos e não residir em Belém.

\section{RESULTADO E DISCUSSÃO}

\subsection{PERGUNTAS}

1. Durante a pandemia, alguma vez, os alimentos acabaram antes que as pessoas da sua família pudessem comprar mais comida?

2. Durante a pandemia, alguma vez, alguém da sua família com 18 anos ou mais não teve uma alimentação saudável e variada devido à redução de condições financeiras?

3. Durante a pandemia, alguém da sua família com 18 anos ou mais, alguma vez, consumiu uma quantidade insuficiente de comida, devido à redução de condições financeiras?

4. Durante a pandemia, alguém da sua família com 18 anos ou mais, alguma vez, sentiu fome, mas não comeu, pois não havia dinheiro para comprar comida?

5. Durante a pandemia, alguém da sua família com menos de 18 anos, alguma vez, não teve uma alimentação saudável e variada devido a redução de condições financeiras?

6. Durante a pandemia, alguém da sua família com menos de 18 anos, alguma vez, consumiu uma quantidade insuficiente de comida devido à redução de condições financeiras?

7. Durante a pandemia, alguém da sua família com menos de 18 anos, alguma vez, sentiu fome, mas não comeu, pois não havia dinheiro para comprar comida?

8. Alguma vez, você deixou de ir comprar alimentos por insegurança de ir ao mercado de forma que isso comprometesse a sua alimentação?

9. Se sua família necessita do Auxílio Emergencial, você considera que isso os ajuda significativamente a manter uma alimentação adequada?

10. Você ou alguém da sua família se encontra no grupo de risco? (idoso, gestante, diabetes, obesidade, cardiopata, asma crônica ou outro tipo de doença que o inclua no grupo de risco) 


\subsection{RESULTADO DAS PERGUNTAS}

Tabela 1 - Resultado das perguntas em percentual

\begin{tabular}{|c|c|c|}
\hline $\mathrm{N}^{\circ}$ das perguntas & SIM & NÃO \\
\hline 1 & $31,6 \%$ & $68,4 \%$ \\
\hline 2 & $32,4 \%$ & $67,6 \%$ \\
\hline 3 & $18,9 \%$ & $81,1 \%$ \\
\hline 4 & $13,5 \%$ & $86,5 \%$ \\
\hline 5 & $5,4 \%$ & $94,6 \%$ \\
\hline 6 & $10,8 \%$ & $89,2 \%$ \\
\hline 7 & $5,4 \%$ & $94,6 \%$ \\
\hline 8 & $29,7 \%$ & $70,3 \%$ \\
\hline 9 & $64,9 \%$ & $35,1 \%$ \\
\hline 10 & $83,8 \%$ & $16,2 \%$ \\
\hline
\end{tabular}

Segundo Aragão (2020), problemáticas como a fome, a falta de acesso regular a alimentos nutritivos, seguros e suficientes, desnutrição, sobrepeso, obesidade, carências nutricionais como deficiencias de vitamina A, D, cálcio, ferro, conhecidas como "fome oculta" já eram incidentes sobre a população brasileira, somados ao desmonte de políticas sociais inclusivas que buscam assegurar a SAN (Segurança Alimentar e Nutricional) contribuiram para aumentar os impactos da pandemia repercutindo no agravamento da insegurança alimentar no país. Além disso, famílias e grupos de vulnerabilidade, principalmente de baixa renda como desempregados e trabalhadores informais estão mais vulneráveis devido à desigualdade social. Nesse sentido, a pandemia trouxe o agravamento das condições socioeconômicas desses grupos devido as medidas adotadas pelo governo como forma de combater a pandemia que impactaram negativamente a renda das famílias com restrições aos trabalhadores informais e redução de vínculos empregatícios diminuindo o acesso aos alimentos.

Dessa forma, os resultados obtidos a respeito da alimentação em maiores de 18 anos demonstra esta problemática que se reflete na aquisição de alimentos, sendo 32,4\% correspondente a não possuir uma alimentação saudável e variada, seguido de 18,9\% não consumir uma quantidade suficiente de comida e $13,5 \%$ de ter sentido fome e não ter se alimentado. Em contrapartida, os resultados obtidos a respeito da alimentação em menores de 18 anos foram menores sendo 5,4\% não possuir uma alimentação saudável e variada, 10,8\% não consumir uma quantidade suficiente de comida e 5,4\% ter sentido fome e não ter se alimentado. Além disso, 31,6\% afirmaram que os alimentos acabaram antes que pudessem comprar mais.

Nesse sentido, segundo Schappo (2021), dentre as diversas medidas para sanar a problemática da fome entre os grupos em maior vulnerabilidade socioeconômica se destaca durante a pandemia a necessidade de fortalecimento da seguridade social, da previdência e assistência social, fortalecimento do Programa Bolsa família e o Auxílio Emergencial. Além disso, outra medida que poderia ser de fundamental importância seria a utilização dos recursos do Pnae (Programa Nacional de Alimentação Escolar) no 
oferecimento de cestas básicas para as famílias dos estudantes priorizando alimentos frescos obtidos da agricultura familiar. Esta medida poderia possibilitar às famílias uma alimentação mais saudável e aos agricultores vinculados ao PNae uma garantia de escoamento da produção e geração de renda, ao contrário do que foi realizado, com a distribuição de cartão de benefício que não garante aos estudantes que tiveram as aulas suspensas uma alimentação saudável, visto que possibilita a aquisão de alimentos ultraprocessados e pouco nutritivos.

Assim, de acordo com os resultados obtidos na pesquisa, 64,9\% afirmaram que o Auxílio Emergencial os ajuda significativamente a manter uma alimentação adequada, portanto, esse resultado confirma a importância das políticas sociais como forma de assegurar a o DHAA (Direito Humano à Alimentação Adequada), principalmente no atual período de pandemia do coronavírus onde grande parte da população teve seus recursos reduzidos, com maior vulnerabilidade das minorias como negros, famílias de baixa renda, idosos, crianças entre outros.

Outro fator importante a ser considerado, segundo Abranches (2020), consiste que grande parte da população como forma de evitar o consumo de alimentos de delivery com potencial contaminação ou pela impossibilidade de adquisição destes necessitam adquirir gêneros alimentícios, itens de limpeza e cuidados pessoais nas redes de mercado, entretanto, a ida ao supermercado pode ser um fator de risco quando este não está preparado para atender à demanda. Em resposta a isso, várias foram as medidas adotadas para minimizar o contágio como a higienização de superfícies com frequência de contato e disponibilização de álcool em gel nas entradas dos estabelecimentos, além disso, foram tomadas medidas que reduziram o acesso aos estabelecimentos, número limitado de clientes no seu interior e separação de horários para idosos. Além disso, as próprias mídias sociais veiculam cenários de caos nesses espaços com falta de cumprimento de medidas de higienização e de cuidados pessoais pelos próprios funcionários bem como aglomerações de pessoas para compra de produtos em excesso. No mais, também destaca sobre a tendência à maior aquisição de alimentos estocáveis em momentos de crise, com maior consumo de alimentos processados e ultraprocessados com medo de uma possível escassez de alimentos, visto que são práticos, de fácil acesso e muitas vezes com menor preço comparados aos alimentos frescos, entretanto, são alimentos muitas vezes de baixo valor nutricional e com excesso em calorias, aditivos, sal e açúcares o que contribui para aumentar a insegurança alimentar nesse período de pandemia em que uma alimentação saudável é imprescindível para a manutenção da saúde e combate ao coronavírus.

Desse modo, o resultado obtido com a pesquisa demonstra que $29,7 \%$ deixaram de ir ao mercado por medo de forma que isso comprometesse a sua alimentação, com 32,4\% dos maiores de 18 anos e 5,4\% dos menores de 18 anos sem uma alimentação saudável e variada o que pode confirmar a associação entre a pandemia e maior aquisição de alimentos processados e ultraprocessados. 
Vale ressaltar, ainda, que que as famílias priorizam as necessidas básicas das crianças e jovens, como demonstrado com os resultados de que 13,5\% maiores de 18 anos e 5,4\% menores de 18 anos sentiram fome mas não comeram. Entretanto, os menores de 18 anos, apesar de terem apresentado nos resultados um percentual menor de não possuir uma alimentação saudável e variada $(5,4 \%)$ em relação aos maiores de 18 anos (32,4\%), podem apresentar uma alimentação igualmente inadequada visto que apesar de serem priorizados no grupo familiar, segundo Costa (2020), durante a pandemia há uma tendência de aumento do consumo de alimentos ultraprocessados nas regiões menos favorecidas Norte e Nordeste, com menor escolaridade e menor faixa etária, deixando em situação de insegurança alimentar os mais jovens. Nesse sentido, o aumento no consumo de alimentos ultraprocessados pode elevar o risco de obesidade, hipertensão e diabetes, cuja presença aumenta a gravidade e a letalidade da covid-19.

Além disso, de acordo com os resultados obtidos 83,4\% afirmaram ter pelo menos um membro da família pertencente ao grupo de risco para a COVID -19, nesse sentido, segundo Daltoé (2020), pessoas idosas e com condições de saúde pré-existentes (hipertensão arterial, diabetes, doenças cardíacas, doenças pulmonares, câncer) estão associadas a desenvolverem doenças graves com maior frequência do que as outras. Desse modo, ressalta para a importância de uma alimentação adequada como forma de previnir a COVID - 19, pois uma nutrição ruim repercute na resposta imune favorecendo a predisposição do indivíduo a infecções, assim, o consumo de ultraprocessados e baixo consumo de vitaminas, minerais e fibras prejudicam significativamente a imunidade adaptativa enquanto aumenta a imunidade inata, levando à inflamação crônica com comprometimento da defesa do organismo à patógenos virais representando um risco maior para o desenvolvimento da forma grave da COVID - 19 em grupos de risco. Assim, verifica se mais uma vez a vulnerabilidade a que os grupos de risco estão sujeitos devido o quadro de insegurança alimentar que já era evidente em boa parte da população, mas que foi agravado com os impactos socioeconômicos ocasionados pela atual pandemia.

\section{CONCLUSÃO}

Tendo em vista as políticas públicas voltadas ao enfrentamento da Covid-19 e seu impacto na sociedade brasileira, vale ressaltar a ineficácia do Governo Federal em responder de forma imediata a emergências. As propostas sugeridas para a amenização dos efeitos da pandemia consistem em aumento de renda da população em situação de vulnerabilidade por meio do Auxílio Emergencial, até a data de publicação deste artigo, ofertando 250 reais ao beneficiário. Contudo, conforme relatado ao longo deste artigo, a insegurança alimentar persiste justamente pelo valor ofertado às famílias de baixa renda ser insuficinente para que todos os membros possam se alimentar de forma satisfatória, auxilia, mas não é o suficiente para suprir a demanda populacional. Nestas situações, notou-se que os integrantes mais velhos cedem seu alimento aos mais jovens. 
Além disto, o receio do contágio na ida à feira/supermercado para obtenção de alimento também é um dos fatores importantes para a persistência da insegurança alimentar, visto que a aglomeração desenfreada aliada ao não cumprimento das normas de segurança por meio dos que frequentam tais lugares, transformam a compra de mantimentos um risco para a saúde das pessoas. Além disso, foi verificado que boa parte de famílias com membros maiores de 18 anos, bem como menores de 18 anos e de grupos de risco possuem algum grau de insegurança alimentar estando vulneráveis, visto a importância da boa alimentação e nutrição no combate à COVID - 19. Assim, é de fundamental importância o conhecimento a respeito do cenário de segurança alimentar da população para que possam ser tomadas medidas que visem combater os flagelos das desigualdades sociais e garantir a segurança alimentar e nutricional como forma de proteger as pessoas da pandemia especialmente as minorias e grupos de risco que tendem a estar mais vulneráveis nesses momentos de crise além de fortalecer as políticas já existentes que visam minimizar tais desigualdades. 


\section{REFERÊNCIAS}

ABRANCHES, Monise Viana; LANA, Raquel Martins. OLIVEIRA, Tatiana Coura. (In) Segurança alimentar no contexto da pandemia por SARS - CoV - 2. Cadernos de Saúde Pública. Paranaíba, 2020.

ALPINO, T. M. A.; SANTOS, C. R. B.; BARROS, D. C.; FREITAS, C. M. COVID-19 e (In)segurança alimentar e nutricional: Ações do Governo Federal brasileiro na pandemia frente aos desmontes orçamentários e institucionais. Caderno de Saúde Pública, v. 36, n. 8, 2020.

ARAGÃO, Érica; BARRETO, Maurício Lima; CAMPELLO, Tereza. Implicações da pandemia COVID - 19 para segurança alimentar e nutricional no Brasil. Revista Ciência e Saúde Coletiva, Salvador, 25 (9), $3421-3430,2020$.

BRASIL, Lei no 11.346, de 15 de setembro de 2006. Cria o Sistema Nacional de Segurança Alimentar e Nutricional - SISAN com vistas em assegurar o direito humano à alimentação adequada e dá outras providências. Brasília (DF); 2006. Artigo 3. [citado 2014 jul 6]. Disponível em: http://www.planalto.gov.br/ccivil_03/_ato2004-2006/2006/Lei/L11346.htm. Acesso em: 10 de Junho de 2021.

COSTA, Caroline dos Santos; GABE, Kamila Tiemman; LEITE, Maria Alvim. Mudanças alimentares na coorte NutriNet Brasil durante a pandemia de Covid - 19. Revista de Saúde Pública, São Paulo, pág. $54-91,2020$.

DALTOÉ, Luciane; DEMOLINER, Fernanda. Covid - 19: Nutrição e comportamento alimentar no contexto da pandemia. Revista Perspectiva: Ciência e Saúde. Osório, V. 5: pág. 36 - 50, ago 2020.

MENEZES, F.; BURITY, V. O novo veto do Capitão da Fome. Le Monde Diplomatique Brasil 2020; 22 mai. Disponível em: https://diplomatique.org.br/o-novo-veto-do-capitao-fome/. Acesso em: 10 de Junho de 2021.

MINUSSI, B. B.; PALUDO, E. A.; PASSOS, J. P. B.; SANTOS, M. J.; MOCELLIN, O.; MAEYAMA, M. A. Grupos de risco do COVID-19: A possível relação entre o acometimento de adultos jovens "saudáveis" e a imunidade. Brazilian Journal of Health Review, Curitiba, v. 3, n. 2, p. 3739-3762 mar./abr. 2020

PICHONELLI, M. Por que você não deve falar "coronavoucher" e estigmatizar mais as pessoas. UOL 2020; 3 abr. Disponível em: https://www.uol.com.br/ecoa/ultimas-noticias/2020/04/03/porquevocenaodevefalarcoronavouchereestigmatizarmaisaspessoas.htm. Acesso em: 10 de Junho de 2021.

PINHEIRO, A. R. O; CARVALHO, M. F. C. C. Transformando o problema da fome em questão alimentar e nutricional: Uma crônica desigualdade social. Revista Ciência e Saúde Coletiva, v. 15, n. 1, jan. 2010.

RIBEIRO-SILVA, R. C.; PEREIRA, M.; CAMPELlO, T.; ARAGÃO, E.; GUIMARÃES, J. M. M.; FERREIRA, A. J. F.; BARRETO, M. L.; SANTOS, S. M. C. Implicações da pandemia COVID-19 para a segurança alimentar e nutricional no Brasil. Revista Ciência e Saúde Coletiva, v. 25, n. 9, set. 2020.

RIGAUD, J. P. O.; VERTHEIN, U. P.; AMPARO-SANTOS, L. Fome em tempos de pandemia de COVID-19: Uma análise crítica aos sentidos (re)produzidos pela mídia. Revista Segurança Alimentar e Nutricional, v. 28, p. 1-13, 2021. 
SANTOS, L. P.; LINDEMANN, I. L.; MOTTA, J. V. S.; MINTEM, G.; BENDER, E.; GIGANTE, D. P. Proposta de versão curta da Escala Brasileira de Insegurança Alimentar. Revista e Saúde Pública, v. 48, n. 5, p. 783-789, 2014.

SCHAPPO, Sirlândia. Fome e insegurança alimentar em tempos de pandemia da Covid - 19. Revista SER Social, Brasília, V. 23, n. 48, janeiro a junho de 2021.

VASCONCELLOS, A. B. P. A.; MOURA, L. B. A. Segurança alimentar e nutricional: uma análise da situação da descentralização de sua política pública nacional. Cadernos de Saúde Pública, v. 34, n. 2, 2018. 